\title{
Sociodemographic and health correlates of sleep problems and duration in older adults in South Africa
}

\author{
K Peltzer \\ HIV/AIDS/SIT and TB (HAST), Human Sciences Research Council, Pretoria, and Department of Psychology, University of Limpopo, Turfloop \\ K Peltzer, $\mathrm{PhD}$
}

Corresponding author: K Peltzer (kpeltzer@hsrc.ac.za)

\begin{abstract}
Objective. To investigate sleeping problems, sleep duration and associated factors in a national probability sample of older South Africans who participated in the Study of Global Ageing and Adult Health (SAGE) in 2008.

Methods. In 2008 I conducted a national population-based cross-sectional study with a sample of 3840 South African individuals aged $\geq 50$ years. A questionnaire was used to survey sociodemographic characteristics and health variables, and anthropometric and blood pressure measurements were recorded.

Results. Of the participants, 9.1\% reported having a sleeping problem at the time of the study. The average number of self-reported hours of sleep was 8.6 ( $\mathrm{SD} \pm 2.1$ ), with $11.6 \%, 45.1 \%, 20.0 \%$, and $23.5 \%$ reporting $\leq 6,7-8,9$, and $\geq 10 \mathrm{~h}$, respectively. In multivariable analysis, depression, cognitive impairment, lack of social cohesion, and moderate or severe activity limitations were associated with having a current sleeping problem. In terms of sociodemographic and health variables, a short sleep duration was associated with: white, Indian/Asian or coloured ethnicity; daily tobacco use; and moderate and severe activity limitations. In participants aged 60 - 79 years, lower wealth, hypertension, risky drinking and lower health-related quality of life were associated with a long sleeping duration.

Conclusion. This study robustly characterised the prevalence of sleeping problems and specific associated risk factors in a large sample in South Africa. This can help to direct future healthcare efforts.
\end{abstract}

S Afr J Psych 2012;18(4):150-156. DOI:10.7196/SAJP.369

A number of studies have associated older age with increased sleep problems $s^{1-3}$ and shorter sleep duration. ${ }^{4-6}$ Overall, socioeconomic factors, emotional support and lifestyle habits have been associated with sleep quality and duration in several populations. ${ }^{3,6}$ It is well documented that poor sleep quality is associated with several adverse health conditions, and this relationship is thought to be bi-directional.,

In a recent study among a large older-aged population of eight low- and middle-income countries from Latin America, India and China, ${ }^{3}$ the prevalence of sleep complaints varied from $9.1 \%$ in China to $37.7 \%$ in India. The average number of self-reported hours of sleep was 7.5 (SD \pm 1.9 ) among older Chinese. ${ }^{6}$ In terms of socioeconomic factors, women tended to have more sleep problems ${ }^{3,6,7}$ and were likely to sleep fewer hours than men. ${ }^{8}$ A lower level of education ${ }^{3,-11}$ and lower income $^{9.11}$ were associated with more reports of sleep complaints. Ethnic or population group differences were also found, e.g. American whites reported more sleep problems than non-whites, ${ }^{12}$ and black Americans were more likely to report both short and long sleep duration than white Americans. ${ }^{13}$ Urban residence was associated with more reports of sleep complaints among older adults in some studies. ${ }^{3,14}$ In terms of health and social factors, sleep complaints were associated with: high pain scores, ${ }^{3}$ poor self-rated health, ${ }^{3,15}$ lower health-related quality of life (QoL), ${ }^{15}$ higher memory impairment score, ${ }^{3}$ major depression, mild cognitive impairment, ${ }^{3}$ lower levels of physical activity, ${ }^{3}$ smoking, ${ }^{10}$ risky alcohol use, and a high number of co-morbidities (hypertension, cardiovascular disease, obesity and other metabolic disorders) ${ }^{3}$ and lower levels of emotional social support or single marital status. ${ }^{17}$ Elders with poorer health status or older age were more likely to have either a relatively shorter $(\leq 6 \mathrm{~h})$ or longer $(\geq 10 \mathrm{~h})$ sleep duration. ${ }^{6}$ In comparison with women who slept $7 \mathrm{~h}$, those with extreme sleep durations $(\leq 5$ or $\geq 10 \mathrm{~h}$ ) reported worse scores on the Short Form Health Survey (SF-36) physical and mental scales. ${ }^{18}$ Factors positively associated with the fifth percentile ( $\leq 4 \mathrm{~h} 30 \mathrm{~min}$ ) of night-time sleep duration were obesity, poor health, insomnia, and insomnia accompanied by daytime sleepiness and cognitive impairment. At the other extremity (95th percentile), long sleep ( $\geq 9$ h $30 \mathrm{~min}$ ) was associated with organic disease, lack of physical exercise and lower education. ${ }^{19}$

I investigated sleeping problems, sleep duration and associated factors in a national probability sample of older South Africans who participated in the Study of Global Ageing and Adult Health (SAGE) in 2008.

\section{Methods}

A national population-based cross-sectional study with a sample of 3840 persons aged $\geq 50$ years was conducted in SA in 2008. The SAGE two-stage probability sample design yielded national and sub-national estimates to an acceptable precision at provincial level, by locality type (urban and rural) and population group (black, coloured, Indian/Asian and white). The first stage of sampling was the selection of primary sampling units (PSUs), using the 2002 Human Sciences Research Council (HSRC) master sample as the sampling frame. The master sample is a probabilistic sample of 1000 enumeration areas (EAs) drawn from the South African National Census, conducted by Statistics South Africa (SSA) in 2001. An EA is the smallest geographical unit allocated to a 
single enumerator during census enumeration. It constitutes a small piece of land for an enumerator to cover to administer a questionnaire during a national population census. The size of most EAs is between 100 and 250 visiting points (VPs). A VP is a separate (non-vacant) residential stand, address, structure or flat in a block of flats or homestead. It is a dwelling and therefore often, but not always, corresponds to a household. For the SAGE study, 600 EAs were drawn from the master sample and used as PSUs. This stage of selection was done centrally at the HSRC. The master sample was stratified by province, residence and race, and the EAs were then selected with a probability proportional to size, with the estimated number of people aged $\geq 50$ years in each EA as a measure of size. Therefore, EAs with a larger number of people aged $\geq 50$ years had a higher chance of being selected.

The second stage of the sample design was the selection of VPs - in this case, households - which formed the secondary sampling units. This stage involved plotting the locations of households on geo-referenced aerial photograph maps of urbanised areas. From these photographs, the co-ordinates of each household in the selected EAs were extracted (using ArcView 3.3 geographical information system software) and used to create a list of households. The household list was updated as necessary after a field visit. Once households had been systematically selected from the updated listing, Garmin eTrex global positioning system (GPS) receivers were used to navigate to the households.

The individual response rate among those aged $\geq 50$ years was $77 \%$. The global SAGE survey was carried out in South Africa in partnership between the National Department of Health (DoH), the HSRC and the World Health Organization (WHO). The study was approved by the HSRC Research Ethics Committee and the National DoH.

\section{Measures \\ Sleeping problems and duration}

The prevalence of sleeping problems was estimated based on the question: 'Overall in the last 30 days, how much of a problem did you have with sleeping, such as falling asleep, waking up frequently during the night, or waking up too early in the morning?' Response options ranged from 1 (none) to 5 (extreme/cannot do). Sleeping problems were defined by the response to this question with 'severe' or 'extreme/cannot do'. The survey also included two questions about self-reported hours of sleep in the preceding two nights: 'How many hours (and minutes) did you sleep?' This was a closed question in which interviewees had to report the number of hours and minutes. Information available did not allow distinction between sleep duration in the night and during the daytime. To capture possible non-linear associations between sleep duration and its associates, we followed a similar categorisation used by previous studies and classified sleep hours into $\leq 6,7-8,9$, and $\geq 10 \mathrm{~h} /$ day. ${ }^{20}$ The category of $7-8 \mathrm{~h}$ /day was used as reference to allow for comparison with earlier studies on older adults. ${ }^{6,20}$ This reference category was chosen because some studies reported that those who slept 7 - 8 h usually had the lowest mortality risk. ${ }^{6,21}$

\section{Depression}

Symptom-based depression in the 12 months preceding recruitment was assessed based on the World Mental Health Survey version of the Composite International Diagnostic Interview. ${ }^{22}$ The diagnosis of depression was based on the ICD-10 Classification of Mental and Behavioural Disorders: Diagnostic Criteria for Research (DCR-10) ${ }^{23}$ and was derived from an algorithm accounting for reported symptoms of depression during the preceding 12 months. ${ }^{24}$ In addition, the ones who responded affirmatively to the question, 'Have you ever been diagnosed with depression?' were added to the symptom-based depression.

\section{Blood pressure}

Systolic blood pressure (SBP) and diastolic blood pressure (DBP) were measured in triplicate on the right arm or wrist of the seated respondent using an automated device. The average of the last 2 readings was used. In accordance with the Seventh Report of the Joint National Committee of Prevention, Detection, Evaluation, and Treatment of High Blood Pressure, ${ }^{25}$ individuals with a SBP $\geq 140 \mathrm{mmHg}$ and/or a DBP $\geq 90 \mathrm{mmHg}$ and/or who reported the current use of anti-hypertensive medication were considered to be suffering from high blood pressure.

\section{Tobacco use}

Lifetime tobacco use was assessed with the question: 'Have you ever smoked tobacco or used smokeless tobacco?'. Lifetime tobacco users were asked: 'Do you currently use (smoke, sniff or chew) any tobacco products such as cigarettes, cigars, pipes, chewing tobacco or snuff?' The response options were: 'yes, daily'; 'yes, but not daily'; and 'no, not at all'. These questions were based on the WHO Guidelines for Controlling and Monitoring the Tobacco Epidemic. ${ }^{26}$

\section{Alcohol use}

Lifetime alcohol use was assessed with the question: 'Have you ever consumed a drink that contains alcohol (such as beer, wine, spirits, etc.)?'. Response options were: 'yes' or 'no, never'. Lifetime alcohol users were asked about current alcohol use in the preceding month, and current alcohol users were asked 'During the past 7 days, how many drinks of any alcoholic beverage did you have each day?' Risky alcohol use was defined as 10 or more alcoholic drinks in the past week.

\section{Cognitive impairment}

Cognitive impairment was defined as severe or extreme difficulty concentrating or remembering things in the past 30 days.

\section{Height and weight}

Body mass index (BMI) - weight (kg) divided by height $(\mathrm{m})$ squared was used as an indicator of obesity (defined as a BMI $\geq 30 \mathrm{~kg} / \mathrm{m}^{2}$ ).

\section{Physical activity}

Physical activity was measured using the General Practice Physical Activity Questionnaire (GPAQ). The GPAQ gathers information on physical activity at work, travel to and from places, and for recreation, as well as on time spent being inactive. It also assesses vigorous and moderate activities performed at work and for recreation. I recorded the number of days per week spent on different activities and time spent in a typical day for each activity. ${ }^{27}$ In addition to the total minutes of activity, the activity volume was computed by weighing each activity type by energy requirement in metabolic equivalents (METs). One MET was defined as the energy cost of sitting quietly, equivalent to a calorie consumption of $1 \mathrm{kcal} / \mathrm{kg} / \mathrm{h}$. An MET minute showed the total activity volume on a weekly basis, calculated by multiplying the time spent on each activity per 


\section{ARTICLE}

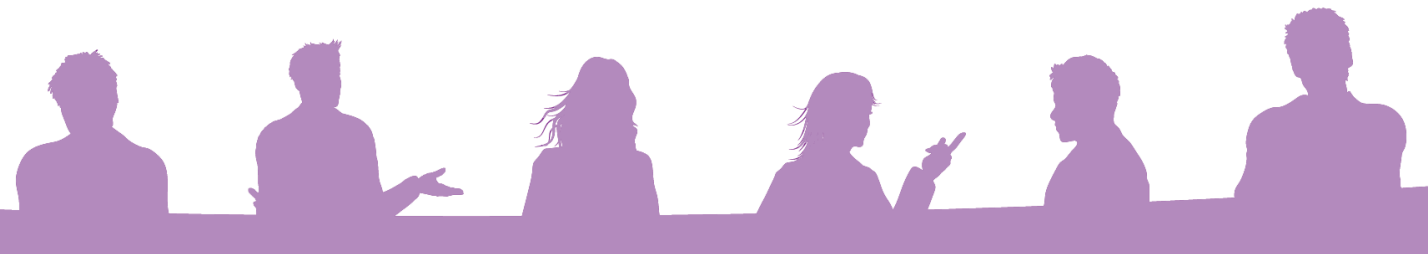

Table 1. Sample characteristics, sleeping problems and sleep duration among older South Africans

\begin{tabular}{|c|c|c|c|c|c|c|}
\hline \multirow[b]{2}{*}{ Variable } & \multirow{2}{*}{$\begin{array}{l}\text { Total sample } \\
N(\%)\end{array}$} & \multirow{2}{*}{$\begin{array}{l}\text { Sleeping problem } \\
\%\end{array}$} & \multicolumn{4}{|c|}{ Sleep duration, $\%$} \\
\hline & & & $\leq 6 \mathrm{~h}$ & $7-8 h$ & $9 \mathrm{~h}$ & $\geq 10 \mathrm{~h}$ \\
\hline All & 3840 & 9.1 & 11.4 & 45.1 & 20.0 & 23.5 \\
\hline \multicolumn{7}{|l|}{ Age (years) } \\
\hline $50-59$ & $1695(49.9)$ & 9.4 & 14.1 & 49.3 & 16.6 & 20.0 \\
\hline $60-69$ & $1233(30.6)$ & 6.9 & 8.2 & 40.5 & 26.8 & 24.5 \\
\hline $70-79$ & $661(14.0)$ & 11.1 & 7.9 & 43.4 & 20.4 & 28.3 \\
\hline$\geq 80$ & $251(5.5)$ & 14.9 & 13.4 & 36.9 & 12.5 & 37.1 \\
\hline \multicolumn{7}{|l|}{ Gender } \\
\hline Male & $1638(44.1)$ & 6.5 & 11.8 & 47.7 & 19.1 & 21.3 \\
\hline Female & $2202(55.9)$ & 11.2 & 11.1 & 43.0 & 20.7 & 25.2 \\
\hline \multicolumn{7}{|l|}{ Population group } \\
\hline Black & $2053(74.0)$ & 9.9 & 8.7 & 41.6 & 20.2 & 29.5 \\
\hline White & $269(9.3)$ & 4.3 & 20.4 & 54.3 & 19.0 & 6.2 \\
\hline Coloured & $655(12.8)$ & 6.2 & 17.1 & 49.6 & 18.9 & 14.4 \\
\hline Indian/Asian & 307 (3.8) & 8.5 & 23.9 & 41.4 & 14.5 & 20.2 \\
\hline \multicolumn{7}{|l|}{ Marital status } \\
\hline Single & $512(14.3)$ & 10.9 & 12.8 & 45.8 & 17.6 & 23.8 \\
\hline Married & 2007 (55.9) & 7.6 & 11.5 & 47.2 & 21.3 & 20.0 \\
\hline Separated/Divorced & $230(5.9)$ & 10.4 & 7.5 & 47.2 & 17.7 & 27.6 \\
\hline Widow & $1020(23.9)$ & 11.1 & 11.7 & 38.7 & 19.1 & 30.5 \\
\hline \multicolumn{7}{|l|}{ Educational level } \\
\hline No schooling & $854(25.2)$ & 9.7 & 8.1 & 36.4 & 22.2 & 33.3 \\
\hline Less than primary & $803(24.0)$ & 10.1 & 11.3 & 36.3 & 20.6 & 31.8 \\
\hline Primary & $779(22.4)$ & 10.9 & 10.8 & 46.7 & 18.8 & 23.6 \\
\hline Secondary or higher & $923(28.3)$ & 4.5 & 14.6 & 53.2 & 17.8 & 14.4 \\
\hline \multicolumn{7}{|l|}{ Wealth } \\
\hline Low & $1482(40.6)$ & 10.1 & 7.0 & 40.3 & 21.4 & 31.3 \\
\hline Medium & $731(18.2)$ & 8.7 & 11.7 & 44.4 & 18.8 & 25.0 \\
\hline High & $1608(41.2)$ & 8.2 & 15.7 & 50.2 & 18.9 & 15.2 \\
\hline \multicolumn{7}{|l|}{ Geolocality } \\
\hline Rural & $1276(35.1)$ & 10.7 & 9.2 & 39.8 & 21.9 & 29.1 \\
\hline Urban & $2561(64.9)$ & 8.3 & 12.6 & 48.0 & 19.0 & 20.4 \\
\hline \multicolumn{7}{|l|}{ Subjective health status } \\
\hline Very good - good & $1469(37.9)$ & 2.3 & 13.1 & 50.8 & 18.0 & 18.1 \\
\hline Moderate & $1681(44.9)$ & 8.5 & 9.9 & 41.6 & 23.3 & 25.3 \\
\hline Bad - very bad & $617(17.5)$ & 26.6 & 12.7 & 37.6 & 17.2 & 32.8 \\
\hline \multicolumn{7}{|l|}{ Other conditions } \\
\hline Arthritis & $851(24.7)$ & 14.5 & 14.3 & 37.8 & 20.8 & 27.1 \\
\hline Hypertension & $2842(77.3)$ & 9.2 & 12.0 & 43.1 & 20.0 & 24.9 \\
\hline Stroke & $139(4.0)$ & 14.8 & 12.8 & 38.0 & 12.2 & 27.1 \\
\hline Angina & $219(5.2)$ & 15.5 & 18.5 & 32.5 & 23.8 & 25.3 \\
\hline Diabetes & $360(9.2)$ & 12.1 & 15.2 & 46.7 & 17.3 & 20.8 \\
\hline Depression & $219(5.8)$ & 33.4 & 17.9 & 35.2 & 19.7 & 27.2 \\
\hline Obese & $1539(46.7)$ & 9.2 & 12.0 & 44.6 & 20.4 & 23.0 \\
\hline Asthma & $165(4.9)$ & 21.3 & 9.1 & 44.1 & 27.5 & 19.3 \\
\hline Cognitive impairment & $263(8.3)$ & 31.5 & 10.0 & 32.3 & 12.6 & 45.0 \\
\hline
\end{tabular}




\section{nor
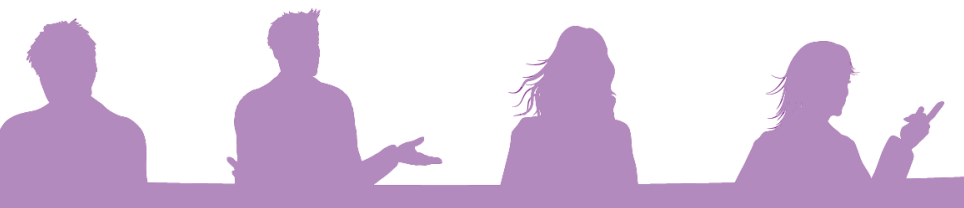

Table 1 (continued). Sample characteristics, sleeping problems and sleep duration among older South Africans

\begin{tabular}{|c|c|c|c|c|c|c|}
\hline \multirow[b]{2}{*}{ Variable } & \multirow{2}{*}{$\begin{array}{l}\text { Total sample } \\
N(\%)\end{array}$} & \multirow{2}{*}{$\begin{array}{l}\text { Sleeping problem } \\
\%\end{array}$} & \multicolumn{4}{|c|}{ Sleep duration, $\%$} \\
\hline & & & $\leq 6 \mathrm{~h}$ & $7-8 h$ & $9 \mathrm{~h}$ & $\geq 10 \mathrm{~h}$ \\
\hline Daily tobacco use & $810(20.4)$ & 12.6 & 15.0 & 41.0 & 19.0 & 25.0 \\
\hline Risky alcohol use ( $\geq 10$ drinks/week) & $158(3.7)$ & 12.0 & 11.0 & 33.5 & 19.4 & 36.1 \\
\hline \multicolumn{7}{|l|}{ Physical activity } \\
\hline Low & $2455(60.5)$ & 9.9 & 9.4 & 43.2 & 22.7 & 24.7 \\
\hline Medium & $446(10.9)$ & 8.2 & 14.4 & 49.6 & 13.9 & 22.0 \\
\hline High & $939(28.6)$ & 7.9 & 14.4 & 47.5 & 16.7 & 21.4 \\
\hline \multicolumn{7}{|l|}{ Activity limitation } \\
\hline None - mild & $2090(55.2)$ & 3.8 & 11.9 & 49.8 & 18.8 & 19.5 \\
\hline Moderate & $1275(34.2)$ & 10.0 & 11.2 & 39.2 & 23.1 & 26.5 \\
\hline Severe - extreme & $370(10.7)$ & 35.7 & 11.2 & 32.2 & 17.9 & 35.7 \\
\hline Social cohesion index (range $9-72)$, mean $( \pm S D)$ & $22.1(6.5)$ & $20.1(6.2)$ & $21.9(6.4)$ & $22.3(7.0)$ & $21.8(5.9)$ & $22.3(6.2)$ \\
\hline QoL (range 0 - 100), mean $( \pm$ SD) & $47.1(12.5)$ & $37(14)$ & $50(14)$ & $49(12)$ & $47(12)$ & $43(12)$ \\
\hline Low & $956(28.5)$ & 19.4 & 10.3 & 34.0 & 20.3 & 24.7 \\
\hline Medium & $1384(33.5)$ & 6.6 & 7.9 & 48.7 & 21.3 & 22.0 \\
\hline High & $1500(38.1)$ & 3.7 & 15.3 & 50.3 & 18.6 & 21.4 \\
\hline
\end{tabular}

week by the MET values of each level of activity. MET values for different activities were set: 4 METs for moderate-intensity physical activity, transport-related walking or cycling; and 8 METs for vigorous physical activity. Total physical activity was calculated as the sum of total moderate, vigorous and transport-related activities per week. Number of days and total physical activity MET minutes per week were used to categorise respondents according to low, moderate, and high levels of activity:

- High - vigorous-intensity activity on $\geq 3$ days, achieving $\geq 1500$ MET $\mathrm{min} /$ week; or $\geq 7$ days of any combination of walking, moderate or vigorous intensity activities, achieving $\geq 3000 \mathrm{MET}$ min/week.

- Moderate - not meeting the criteria for 'high' activity, but meeting any of: $\geq 3$ days of vigorous-intensity activity of $\geq 20 \mathrm{~min} /$ day; $\geq 5$ days of moderate-intensity activity or walking of $\geq 30 \mathrm{~min} /$ day; or $\geq 5$ days of any combination of walking, moderate or vigorous intensity activities achieving $\geq 600 \mathrm{MET} \mathrm{min} /$ week.

- Low - not meeting criteria for high or moderate activity, including physical inactivity.

Moderate and high levels of physical activity were collapsed in further analysis. ${ }^{27}$

\section{Social cohesion}

Social cohesion was measured by asking respondents how often they had engaged in 9 activities in the preceding 12 months (e.g. attended any group, club, society, union or organisational meeting). Response options ranged from 1 (never) to 5 (daily). Cronbach's alpha for this social cohesion index in the sample was 0.73 .

\section{QoL}

QoL was assessed with the WHOQol-8 instrument containing 8 items empirically derived from the WHOQOL-Bref. ${ }^{28}$ The summative model was used producing an index. Cronbach's alpha for the WHOQol-8 was 0.85 in this sample.

\section{Economic status}

A random-effects probit model was used to identify indicator-specific thresholds, representing the point on the wealth scale above which households are likelier to own a given asset than not, and enabling estimation of an asset ladder. These threshold estimates, combined with actual asset ownership for given households, were used for an estimate household-level wealth, which was used to create wealth quintiles. ${ }^{29}$

\section{Chronic conditions}

Other chronic conditions such as arthritis, stroke, angina, asthma, chronic lung disease and diabetes were assessed by self-report.

\section{Data analysis}

The data, captured with CSPro and analysed with STATA software (version 10), were weighted using post-stratified individual probability weights based on the selection probability at each stage of selection. Individual weights were post-stratified by province, sex and age, according to the 2009 SSA medium mid-year population estimates (http://www.statssa.gov.za/publications/P0302/P03022009.pdf). Weights were not normalised. Outliers were removed after boxplot analyses. Computed estimates and odds ratios (ORs) were reported with $95 \%$ confidence intervals (CIs). A double-sided $p$-value of 0.05 used as the cut-off for statistical significance. Associations between key outcomes of overall cognition and sociodemographic, social and health variables were evaluated with ORs. Unconditional multivariable logistic regression was used to evaluate the effect of explanatory variables on the key outcome of sleeping problems (binary-dependent variable). All variables statistically significant at $p<0.05$ in bivariate analyses were included in the multivariable models. Here weighted percentages are reported. The sample size refers to the number of participants asked the target question. The reported 95\% CIs and $p$-values were adjusted for the multi-stage stratified-cluster sample design. Multinomial logistic regression analysis was used to estimate associations between 
Table 2. Multivariable logistic regression with sleeping problem in older South Africans

\begin{tabular}{lll}
\hline & UOR $(95 \%$ CI $)$ & AOR $(\% \%$ CI $)$ \\
\hline $\begin{array}{ll}\text { Gender } \\
\text { Female }\end{array}$ & 1.00 & 1.00 \\
$\quad$ Male & $0.56(0.38-0.82)^{\dagger}$ & $0.64(0.41-1.00)$ \\
Age (years) & & \\
$50-59$ & 1.00 & 1.00 \\
$60-69$ & $0.71(0.46-1.12)$ & $0.50(0.21-1.02)$ \\
$70-79$ & $1.20(0.75-1.93)$ & $1.03(0.57-1.44)$ \\
$\geq 80$ & $1.69(0.92-3.10)$ & $1.21(0.64-2.56)$
\end{tabular}

Population group

Black $\quad 1.00$

White

$0.41(0.15-1.16)$

Coloured

$0.60(0.34-1.06)$

Indian/Asian

$0.84(0.39-1.80)$

Marital status

Single $\quad 1.00$

Married

$0.68(0.33-1.39)$

Separated/Divorced

$0.95(0.42-2.17)$

Widow

$1.02(0.49-2.15)$

Educational level

Wealth

Geolocality

Rural

Urban
No schooling

1.00

Less than primary

$0.94(0.63-1.40)$

Primary

Secondary or higher

$1.02(0.60-1.74)$

$0.40(0.18-0.87)^{*}$

Low

Medium

1.00

High

$0.84(0.57-1.25)$

$0.79(0.54-1.15)$

1.00

$0.76(0.41-1.41)$

Other conditions

Arthritis

Hypertension

Stroke

Angina

Diabetes

Depression

Obese

Asthma

Chronic lung disease

$2.06(0.95-4.44)$

$0.98(0.73-1.31)$

$1.72(0.68-4.35)$

$1.86(0.94-3.67)$

$1.39(0.83-2.32)$

$5.87(3.26-10.56)^{\ddagger}$

$1.01(0.70-1.45)$

$2.83(1.21-6.62)^{*}$

$3.03(1.03-8.92)^{*}$

Cognitive impairment

$5.89(3.93-8.82)^{\ddagger}$

$1.55(1.01-2.40)^{*}$

$1.34(0.69-2.58)$

1.00

0.78 (0.51 - 1.19)

$0.97(0.54-1.75)$

$0.53(0.24-1.20)$ ( $\geq 10$ drinks/week)

$2.23(1.08-4.62)$

$1.42(0.50-4.04)$

$2.43(1.44-4.10)^{\dagger}$

$1.49(0.95-2.34)$

$-\cdot$
Table 2 (continued). Multivariable logistic regression with sleeping problem in older South Africans (continued)

\begin{tabular}{|c|c|c|}
\hline & UOR (95\% CI) & AOR (\%\% CI) \\
\hline \multicolumn{3}{|l|}{ Physical activity } \\
\hline Low & 1.00 & --- \\
\hline Moderate & $0.81(0.40-1.64)$ & \\
\hline High & $0.77(0.41-1.46)$ & \\
\hline Social cohesion index & $0.94(0.91-0.98)^{\ddagger}$ & $0.96(0.93-0.99)^{*}$ \\
\hline \multicolumn{3}{|l|}{ Activity limitation } \\
\hline None - mild & 1.00 & 1.00 \\
\hline Moderate & $2.79(1.91-4.07)^{\ddagger}$ & $2.01(1.33-3.03)^{\ddagger}$ \\
\hline Severe - extreme & $13.95(8.00-24.32)^{\ddagger}$ & $7.76(4.47-13.47)^{\ddagger}$ \\
\hline $\begin{array}{l}\text { UOR }=\text { unadjusted odds ratio } \\
{ }^{*} p<0.5 \\
{ }^{+} p<0.01 . \\
{ }^{*} p<0.001 .\end{array}$ & ljusted odds ratio. & \\
\hline
\end{tabular}

sleep duration and other variables. The multinomial logistic model is a generalisation of the logistic model and has the same function of canonical connection (logistic function). Its use is appropriate when the response variable is categorical and polytomic, the categories of which are mutually exclusive and have no implicit order between them. ${ }^{30}$ In this analysis, the sleep duration was simultaneously compared with the independent reference category ( 7 - $8 \mathrm{~h}$ of sleep). To reduce possible bias in our data analysis and inference due to missing items (although only about $2.5 \%$ of the data are missing for key variables), I imputed missing values with the variable mean for continuous variables, and with the mode of the variable for categorical variables.

\section{Results}

\section{Sample characteristics and sleeping problem and duration}

The total sample included 3840 South Africans aged $\geq 50$ years; $44.1 \%$ men and $55.9 \%$ women. The most prevalent population group was black (74\%); and $49.9 \%$ were aged 50 - 59 years. The education of most participants (71.6\%) was lower than secondary school level and almost two-thirds (64.9\%) lived in an urban area. A very large proportion had hypertension (77.3\%), 24.7\% arthritis, 5.8\% depression combining self-ported diagnosed depression and symptom reporting, 9.2\% had diabetes, $8.3 \%$ were cognitively impaired and $46.7 \%$ were obese. In addition, $4.0 \%$ had had a stroke, $5.2 \%$ angina, $4.9 \%$ asthma and $2.9 \%$ chronic lung disease. Over half $(60.5 \%)$ engaged in low physical activity, $20.4 \%$ were daily tobacco users, and $3.7 \%$ were risky alcohol users. The mean social cohesion score was 22.1 , and $38.1 \%$ rated their QoL as high. Almost 1/10 (9.1\%) participants reported a current sleeping problem. The average number of self-reported hours of sleep was 8.6 (SD \pm 2.1 ), with $11.6 \%, 45.1 \%, 20.0 \%$, and $23.5 \%$ of participants reporting $\leq 6,7-8,9$, and $\geq 10 \mathrm{~h}$ (weighted), respectively (Table 1 ).

\section{Predictors of sleeping problem}

In univariate analysis, being female, not having secondary education or higher, depression, asthma, chronic lung disease, cognitive impairment, daily or almost daily tobacco use, lack of social cohesion and moderate or severe activity limitations were associated with having a current sleeping problem. In multivariable analysis, depression, cognitive impairment, 
Table 3. Multinomial logistic regression with sleeping duration and sociodemographics in older South Africans

\begin{tabular}{|c|c|c|}
\hline & \multicolumn{2}{|c|}{ ARRR1 (95\% CI) } \\
\hline & $\leq 6 \mathrm{~h}(\mathrm{v} .7-8 \mathrm{~h})$ & $\geq 9$ h $($ v. $7-8$ h $)$ \\
\hline \multicolumn{3}{|l|}{ Gender } \\
\hline Female & 1.00 & 1.00 \\
\hline Male & $1.30(0.85-2.01)$ & $1.00(0.67-1.49)$ \\
\hline \multicolumn{3}{|l|}{ Age (years) } \\
\hline $50-59$ & 1.00 & 1.00 \\
\hline $60-69$ & $0.53(0.27-1.05)$ & $1.61(1.10-2.37)^{*}$ \\
\hline $70-79$ & $0.56(0.24-1.29)$ & $1.59(1.14-2.22)^{\dagger}$ \\
\hline$\geq 80$ & $1.51(0.47-4.88)$ & $1.52(0.70-3.36)$ \\
\hline \multicolumn{3}{|l|}{ Population group } \\
\hline Black & 1.00 & 1.00 \\
\hline White & $2.43(1.23-4.82)^{*}$ & $0.55(0.22-1.37)$ \\
\hline Coloured & $1.82(1.02-3.25)^{*}$ & $0.67(0.41-1.08)$ \\
\hline Indian/Asian & $2.37(1.40-4.00)^{\dagger}$ & $0.74(0.53-1.04)$ \\
\hline \multicolumn{3}{|l|}{ Marital status } \\
\hline Single & 1.00 & 1.00 \\
\hline Married & $0.66(0.25-1.76)$ & $1.30(0.60-2.84)$ \\
\hline Separated/Divorced & $0.43(0.15-1.25)$ & $0.99(0.43-2.24)$ \\
\hline Widow & $1.00(0.32-3.13)$ & $1.29(0.53-3.13)$ \\
\hline \multicolumn{3}{|l|}{ Educational level } \\
\hline No schooling & 1.00 & 1.00 \\
\hline Less than primary & $1.69(0.85-3.34)$ & $1.14(0.72-1.81)$ \\
\hline Primary & $1.41(0.79-2.51)$ & $0.98(0.61-1.57)$ \\
\hline Secondary or more & $1.13(0.57-2.22)$ & $0.82(0.54-1.52)$ \\
\hline \multicolumn{3}{|l|}{ Wealth } \\
\hline Low & 1.00 & 1.00 \\
\hline Medium & $1.21(0.60-2.46)$ & $0.59(0.41-0.85)^{\dagger}$ \\
\hline High & $1.31(0.70-2.51)$ & $0.50(0.35-0.72)^{\ddagger}$ \\
\hline \multicolumn{3}{|l|}{ Geolocality } \\
\hline Rural & 1.00 & 1.00 \\
\hline Urban & $0.65(0.34-1.22)$ & $0.89(0.68-1.15)$ \\
\hline \multicolumn{3}{|c|}{$\begin{array}{l}\text { ARRR }=\text { adjusted relative risk ratio. } \\
{ }^{*} p<0.5 \text {. } \\
\dagger p<0.01 \\
\ddagger p<0.001 .\end{array}$} \\
\hline
\end{tabular}

lack of social cohesion, and moderate or severe activity limitations were associated with having a current sleeping problem (Table 2).

\section{Predictors of short and long sleep duration}

In terms of sociodemographic variables, white, coloured or Indian/ Asian ethnicity was associated with short sleep duration. An age of $60-79$ years and lower wealth were associated with long sleep duration (Table 3). In terms of health variables, daily tobacco use and moderate and severe activity limitations were associated with short sleep duration, and hypertension, being a risky drinker and lower health-related QoL were associated with long sleep duration (Table 4).

\begin{tabular}{|c|c|c|}
\hline & \multicolumn{2}{|c|}{ ARRR (95\% CI) } \\
\hline & $\leq 6 h(v .7-8 h)$ & $\geq 9$ h (v. $7-8$ h) \\
\hline \multicolumn{3}{|l|}{ Subjective health status } \\
\hline Very good - good & 1.00 & 1.00 \\
\hline Moderate & $0.87(0.50-1.40)$ & $1.18(0.85-1.85)$ \\
\hline Bad - very bad & $0.63(0.26-1.53)$ & $0.78(0.45-1.37)$ \\
\hline Sleeping problem & $1.54(0.47-5.05)$ & $0.69(0.31-1.51)$ \\
\hline Arthritis & $0.89(0.56-1.42)$ & $1.21(0.91-1.62)$ \\
\hline Hypertension & $1.10(0.62-1.95)$ & $1.28(1.00-1.63)^{*}$ \\
\hline Stroke & $1.06(0.20-5.61)$ & $1.00(0.54-1.86)$ \\
\hline Angina & $1.75(0.68-4.50)$ & $1.28(0.86-1.90)$ \\
\hline Diabetes & $1.08(0.58-4.50)$ & $0.82(0.51-1.30)$ \\
\hline Depression & $1.13(0.52-2.46)$ & $0.85(0.45-1.60)$ \\
\hline Obese & $1.10(0.68-1.79)$ & $0.89(0.72-1.10)$ \\
\hline Asthma & $1.00(0.49-2.07)$ & $1.24(0.62-2.46)$ \\
\hline Chronic lung disease & $1.48(0.29-7.61)$ & $1.31(0.56-3.05)$ \\
\hline Cognitive impairment & $0.74(0.30-1.79)$ & $1.12(0.74-1.67)$ \\
\hline Daily tobacco use & $1.89(1.08-3.31)^{*}$ & $1.21(0.93-1.56)$ \\
\hline $\begin{array}{l}\text { Risky alcohol use } \\
\text { ( } \geq 10 \text { drinks/week) }\end{array}$ & $1.49(0.50-4.43)$ & $2.69(1.07-6.73)^{*}$ \\
\hline \multicolumn{3}{|l|}{ Physical activity } \\
\hline Low & 1.00 & 1.00 \\
\hline Moderate & $1.39(0.73-2.65)$ & $0.70(0.45-1.08)$ \\
\hline High & $1.30(0.67-2.54)$ & $0.74(0.53-1.04)$ \\
\hline \multicolumn{3}{|l|}{ Activity limitation } \\
\hline None - mild & 1.00 & 1.00 \\
\hline Moderate & $1.81(1.09-3.00)^{*}$ & $1.29(0.79-2.09)$ \\
\hline Severe - extreme & $3.06(1.64-5.69)^{\dagger}$ & $1.79(0.88-3.62)$ \\
\hline \multicolumn{3}{|l|}{ QoL } \\
\hline Low & 1.00 & 1.00 \\
\hline Medium & $0.55(0.29-1.07)$ & $0.89(0.60-1.34)$ \\
\hline High & $0.90(0.40-1.99)$ & $0.62(0.39-0.99)^{*}$ \\
\hline \multicolumn{3}{|c|}{$\begin{array}{l}\text { ARRR }=\text { adjusted relative risk ratio; } \mathrm{QoL}=\text { quality of life. } \\
\text { Variables included age, gender, education, marital status and wealth. } \\
{ }^{*} p<0.5 \text {. } \\
\dagger p<0.001 .\end{array}$} \\
\hline
\end{tabular}

\section{Discussion}

The overall prevalence of current sleeping problems in this national sample of older South Africans was 9.1\%. These rates are similar to that found in other studies in low- and middle-income countries, ${ }^{3}$ including South Korea and Taiwan, ${ }^{32}$ but lower than in a large study of INDEPTH sites from eight Asian and African countries (16.6\%). The average number of self-reported hours of sleep was 8.6, with $11.6 \%, 45.1 \%, 20.0 \%$, and $23.5 \%$ reporting $\leq 6,7-8,9$, and $\geq 10 \mathrm{~h}$, respectively. This duration seemed longer than that found in other studies, with a mean of $7-7.5 \mathrm{~h}$ and a larger group $(\geq 30 \%)$ with short sleep duration. ${ }^{6,19}$ 
Consistent with other studies, ${ }^{3,17}$ depression, cognitive impairment, lack of social cohesion and moderate or severe activity limitations were associated with a current sleeping problem. Depression has been found in several studies to be a risk factor for sleep disturbances. ${ }^{33,34}$ Unlike in certain other studies, ${ }^{3,6,79-12,14}$ gender, age, socioeconomic status, population group, urban residence, health behaviour (tobacco use, alcohol use and physical activity) and chronic conditions were not found to be associated with sleeping problems.

In terms of sociodemographic and health variables, white, Indian/ Asian and coloured ethnicity, daily tobacco use and moderate and severe activity limitations were associated with short sleep duration. Among participants aged 60 - 79 years, lower wealth, hypertension, being a risky drinker and lower health-related QoL were associated with long sleep duration. Other studies also found lower healthrelated QoL to be associated long sleep durations. ${ }^{18}$ Several large studies have associated both short and long average sleep durations with an increased prevalence of hypertension, particularly at the extreme of less than $6 \mathrm{~h} /$ night in adults, ${ }^{35}$ but in other studies this was not the case. ${ }^{36}$ Unlike other studies, ${ }^{6,19}$ in this study, poorer health status, obesity, insomnia, organic disease, lack of physical exercise and lower education were not associated with a relatively shorter or longer sleep duration.

\section{Study limitations}

This study had several limitations. Firstly, the self-report of health variables such as sleeping problem and duration, depression symptoms, tobacco or alcohol use should be interpreted with caution. The characterisation of sleeping problems was based on one broad-sense question and, therefore, the influence of insomnia, excessive daytime sleepiness, sleep-disordered breathing and movement disorder could not be evaluated. ${ }^{3}$ These broad-sense criteria might have overestimated the prevalence of sleeping problems, as found previously. ${ }^{37}$ Finally, as the data were collected in a cross-sectional survey, we cannot ascribe causality to any of the associated factors. There is an urgent need to conduct longitudinal studies to investigate the causes of poor health and how it is mediated by poor sleep quality in middle-income countries.

\section{Conclusion}

There was a significant prevalence of sleeping problems in the sample of older adults in SA. Potential factors associated with the risk for reporting sleeping complaints and extreme sleep duration were related to health, and sociodemographic and lifestyle factors. These findings may assist in prevention strategies to promote a better quality of sleep and subsequent QoL for this population.

Acknowledgment. Funding was provided by the DoH. Additional funding was obtained from the United States National Institute on Aging through an interagency agreement with WHO and the HSRC.

\section{References}

1. Roepke SK, Ancoli-Israel S. Sleep disorders in the elderly. Indian J Med Res 2010;131:302-310. IP: 41.243.238.149.

2. Crowley K. Sleep and sleep disorders in older adults. Neuropsychol Rev 2011;21:41-53. [http:// dx.doi.org/10.1007/s11065-010-9154-6]

3. Mazzotti DR, Guindalini C, Sosa AL, Ferri CP, Tufik S. Prevalence and correlates for sleep complaints in older adults in low and middle income countries: A 10/66 Dementia Research Group study. Sleep Med 2012; 13:697-702. [http://dx.doi.org/10.1016/j.sleep.2012.02.009]
4. Hale L. Who has time to sleep? J Public Health 2005;27:205-211. [http://dx.doi.org/10.1093/ pubmed/fdi004]

5. Patel SR, Malhotra A, Gottlieb DJ, White DP, Hu FB. Correlates of long sleep duration. Sleep 2006;29:881-889.

6. Gu D, Sautter J, Pipkin R, Zeng Y. Sociodemographic and health correlates of sleep quality and duration among very old Chinese. Sleep 2010;33(5):601-610.

7. Santos-Silva R, Bittencourt LR, Pires ML, de Mello MT, Taddei JA, Benedito-Silva AA, et al. Increasing trends of sleep complaints in the city of Sao Paulo, Brazil. Sleep Med 2010;11:520-524. [http://dx.doi.org/10.1016/j.sleep.2009.12.011]

8. Moore JP, Adler NE, Williams DR, Jackson JS. Socioeconomic status and health: The role of sleep Psychosom Med 2002;64:337-344. [http://dx.doi.org/10.0033-3174/02/6402-0337]

9. Krueger PM, Friedman EM. Sleep duration in the United States: a cross-sectional population-based study. Am J Epidemiol 2009;169:1052-1063. [http://dx.doi.org/10.1093/aje/kwp023]

10. Foley D, Ancoli-Israel S, Britz P, Walsh J. Sleep disturbances and chronic disease in older adults: results of the 2003 National Sleep Foundation Sleep in America Survey. J Psychosom Res 2004;56:497-502. [http://dx.doi.org/10.1016/j.jpsychores.2004.02.010]

11. Su TP, Huang SR, Chou P. Prevalence and risk factors of insomnia in community-dwelling Chines elderly: A Taiwanese urban area survey. Aust N Z J Psychiatry 2004;38:706-713. [http://dx.doi org/10.1080/j.1440-1614.2004.01444.x]

12. Jean-Louis G, Magai CM, Cohen CI, et al. Ethnic differences in self-reported sleep problems in older adults. Sleep 2001;24:926-933

13. Hale L, Phuong Do D. Racial differences in self-reports of sleep duration in a population-based study. Sleep 2007;30:1096-1103.

14. Hays JC, Blazer DG, Foley DJ. Risk of napping: excessive daytime sleepiness and mortality in an older community population. J Am Geriatr Soc 1996;44:693-698.

15. Magee CA, Caputi P, Iverson DC. Relationships between self-rated health, quality of life and sleep duration in middle aged and elderly Australians. Sleep Med 2011;12:346-350. [http://dx.doi. org/10.1016/j.sleep.2010.09.013]

16. National Institutes of Health. State of the Science Conference: Statement on Manifestations and Management of Chronic Insomnia in Adults, 13 - 15 June 2005. Sleep 2005;28:1049-1057.

17. Liu X, Liu L. Sleep habits and insomnia in a sample of elderly persons in China. Sleep 2005;28:1579-1587.

18. Faubel R, Lopez-Garcia E, Guallar-Castillón P, Balboa-Castillo T, Gutiérrez-Fisac JL, Banegas JR, Rodríguez-Artalejo F. Sleep duration and health-related quality of life among older adults: a population-based cohort in Spain. Sleep 2009;32(8):1059-1068.

19. Ohayon MM, Vecchierini MF. Normative sleep data, cognitive function and daily living activities in older adults in the community. Sleep 2005;28(8):981-989.

20. Lima MG, Bergamo Francisco PM, de Azevedo Barros MB. Sleep duration pattern and chronic diseases in Brazilian adults (ISACAMP, 2008/09). Sleep Med 2012;13(2):139-144. [http://dx.doi org/10.1016/j.sleep.2011.07.011]

21. Hublin C, Partinen M, Koskenvuo M, Kaprio J. Sleep and mortality: A population-based 22 year follow-up study. Sleep 2007;30:1245-1253.

22. Kessler RC, Ustun TB. The World Mental Health (WMH) Survey Initiative Version of the World Health Organization (WHO) Composite International Diagnostic Interview (CIDI). Intern J Meth Psychiatric Res 2004;13:93-121.

23. World Health Organisation (WHO). The ICD-10 Classification of Mental and Behavioural Disorders: Diagnostic Criteria for Research (DCR-10). Geneva: WHO, 1993.

24. Ayuso-Mateos JL, Nuevo R, Verdes E, Naidoo N, Chatterji S. From depressive symptoms to depressive disorders: the relevance of thresholds. Br J Psychiatry 2010;196(5):365-371. [http:// dx.doi.org/10.1192/bjp.bp.109.071191]

25. Chobanian AV, Bakris GL, Black HR, Cushman WC, Green LA, Izzo JL Jr, et al. Seventh report o the Joint National Committee of Prevention, Detection, Evaluation, and Treatment of High Blood Pressure. JAMA 2003;289(19):2560-2572.

26. World Health Organization (WHO). Guidelines for Controlling and Monitoring the Tobacco Epidemic. Geneva, Switzerland: WHO, 1998

27. World Health Organization (WHO). Global Physical Activity Surveillance. Geneva: WHO, 2009 http://www.who.int/chp/steps/GPAQ/en/index.html (accessed 2 December 2010).

28. Power M. Development of a Common Instrument for Quality of Life. In: Nosikov A, Gudex C. EUROHIS: Developing Common Instruments for Health Surveys. Amsterdam: IOS Press, 2003:57:145-163.

29. Chatterji S, Kowal P, Mathers C, et al. The health of aging populations in China and India. Health Aff (Millwood) 2008;27(4):1052-1063. [http://dx.doi.org/10.1377/hlthaff.27.4.1052]

30. Agresti A. Categorical Data Analysis. New York: John Wiley; 1990.

31. Winship C, Radbill L. Sampling weights and regression analysis. Sociol Methods Res 1994;23:230-257.

32. Nomura K, Yamaoka K, Nakao M, Yano E. Social determinants of self-reported sleep problem in South Korea and Taiwan. J Psychosom Res 2010;69:435-440. [http://dx.doi.org/10.1016/j. jpsychores.2010.04.014

33. Stranges S, Tigbe W, Gómez-Olivé FX, Thorogood M, Kandala NB. Sleep problems: An emerging global epidemic? Findings from the INDEPTH WHO-SAGE study among more than 40,000 older adults from 8 countries across Africa and Asia. Sleep 2012;35(8):1173-1181.

34. Rodin J, McAvay G, Timko C. A longitudinal study of depressed mood and sleep disturbances in elderly adults. J Gerontol 1988;43:45-53. [http://dx.doi.org/10.1093/geronj/43.2.P45]

35. Gottlieb DJ, Redline S, Nieto FJ, et al. Association of usual sleep duration with hypertension: The Sleep Heart Health Study. Sleep 2006;29:1009-1014.

36. Van den Berg JF, Tulen JH, Neven AK, et al. Sleep duration and hypertension are not associated in the elderly. Hypertension 2007;50(3):585-589. [http://dx.doi.org/10.1161/hypertensionaha.107.092585]

37. Fok M, Stewart R, Besset A, Ritchie K, Prince M. Incidence and persistence of sleep complaints in a community older population. Int J Geriatr Psychiatry 2010;25:37-45. [http://dx.doi.org/10.1002/ gps.2295] 